\title{
A localization method for loose parts monitoring system of VVER reactor plants*
}

\author{
Ivan V. Maksimov ${ }^{1}$, Vladimir V. Perevezentsev² \\ 1 “KVANT PROGRAMM" LTD, 5/12 Zeleny prospect, Moscow, 111558 Russia \\ 2 Bauman Moscow State Technical University, 5/1 2-nd Baumanskaya str., Moscow, 105005 Russia \\ Corresponding author: Ivan V. Maksimov (iv_maksimov@mail.ru)
}

Academic editor: Boris Balakin • Received 30 October 2019 • Accepted 10 January 2020 • Published 27 March 2020

Citation: Maksimov IV, Perevezentsev VV (2020) A localization method for loose parts monitoring system of VVER reactor plants. Nuclear Energy and Technology 6(1): 29-35. https://doi.org/10.3897/nucet.6.51252

\begin{abstract}
As operational experience shows, it can hardly be excluded that some detached or loosened parts and even foreign objects (hereinafter referred to as the 'loose parts') may appear in the main circulation loop of VVER reactor plants. Naturally, the sooner such incidents are detected and evaluated, the more time will be available to eliminate or at least minimize damage to the reactor plant main equipment. The paper describes a method for localizing the impact of loose parts located in the coolant circulation circuit of a VVER reactor plant. To diagnose malfunctions of the reactor plant main equipment, it is necessary to accurately determine the place where the acoustic anomaly occurred. Therefore, if some loose parts make themselves felt, it is important to track the path of their movement along the main circulation circuit as well as their location using physical barriers.

The method is based on the representation of the surface, along which an acoustic wave travels, as a 3D model of the reactor plant (RP) main circulation circuit. The model has the form of a graph in which the vertices characterize the control points on the RP surface and the edges are the distances between them. The method uses information about the acoustic wave velocity and the time difference of arrivals (TDOAs) of the signal received by various sensors. It is shown that, when the effect is received by more than three sensors, along with an estimate of the impact coordinate, it becomes possible to estimate the average acoustic wave velocity. To determine time of arrival, the signal dispersion change point detection method is used. Provided that the average size between the control points on the RP surface was $300 \mathrm{~mm}$, the average localization error was about $600 \mathrm{~mm}$. The developed algorithm can be easily adapted to any VVER reactor plant. The obtained deviation values are acceptable for practical use.
\end{abstract}

\section{Keywords}

Loose Parts Monitoring System (LPMS), diagnostics of NPPs, impact localization, acoustic wave, main circulation circuit, VVER reactor plant

\section{Introduction}

One of the main condition monitoring systems of VVER reactor plants is the Loose Parts Monitoring System
(LPMS), which function is detecting and evaluating the parameters of loose parts in the coolant circulation circuit. Loose parts can move with the coolant flow in the circulation circuit and cause damage to the RP primary

* Russian text published: Izvestiya vuzov. Yadernaya Energetika (ISSN 0204-3327), 2019, n. 4, pp. 28-38. 
circuit equipment. They can to some extent block the coolant flow, resulting in deteriorated heat transfer, possible overheating of the fuel element claddings, changes in the fuel temperature, and increased fuel swelling rate. Another negative consequence may be the risk of these parts falling into the movable mechanisms of the control and protection system working bodies. In case of their untimely detection in the coolant circulation circuit, the costs of measures taken to eliminate the damage caused by them increase significantly.

Currently, most NPPs with pressurized water reactors (PWR, VVER) are equipped with LPMSs. There are several international standards defining the requirements that LPMSs must meet:

- U.S. Nuclear Regulatory Commission (NRC) Regulatory Guide 1.133 (1981) (Regulatory Guide 1.133 1981);

- American Society of Mechanical Engineers (ASME) Standard OM-2017 (Operation and Maintenance 2015);

- International Electrotechnical Commission (IEC) Standard IEC-609887 (IEC 60988 Nuclear power plants 2009).

Localizing the impact source is one of the most important functions of the LPMS when diagnosing the RP equipment (ISO 13379-1-2015 2015). To diagnose malfunctions of the RP main equipment, it is necessary to accurately determine the place where the acoustic anomaly occurred. Therefore, if some loose parts appear, it is important to track the path of their movement along the main circulation circuit as well as their location.

To date, a large number of studies on impact source localization methods have been performed (Olma 1985, Szappanos et al. 1999, Kim Jung Soo et al. 2003, Choi et al. 2011, Park and Kim 2006, Park Gee Yong et al. 2006, Ki HI et al. 2017, Figedy and Oksa 2005, Liska and Kunkel 2017, Zheng et al. 2008). The traditional and most trivial method localizes the source of the acoustic anomaly to the equipment controlled by the sensor that first detected the impact. If there is a registered effect in more than one sensor, the source is localized using TDOAs of the wave in two or more measuring sensors.

One of the most well-known methods using TDOAs is the method of intersecting hyperbolas (Olma 1985, Park and Kim 2006), which is carried out in manual mode and requires additional expert knowledge from the system operator. The algorithm has a simple analytical solution for the plane, but it is not adapted for the complex surface of the RP primary circuit.

Another method (Szappanos et al. 1999, Figedy and Oksa 2005, Liska and Kunkel 2017) is based on the search for the closest match to the recorded TDOAs calculated earlier. To do this, the primary circuit is divided into segments of a small area or control points. Given that the velocity of acoustic waves in metal is known, it is possible to pre-calculate the wave propagation time from individual segments to the sensors. The obtained TDOA values are stored in the database. When an acoustic anomaly is recorded, its location is selected from the database as the nearest stored vector to the measured one in accordance with the Euclidean distance. In (Liska and Kunkel 2017), the errors of the localization method and its structural limitations are analyzed. To determine the time of arrival, it is necessary to know the acoustic wave velocity. Since it depends on the material, kinetic energy of interaction, mass and shape of loose parts, as well as other parameters, this method introduces great uncertainty in assessing the acoustic anomaly source localization.

The proposed algorithm automatically performs localization to a point on the surface of the RP primary circuit. It is based on calculating the shortest path along a $3 \mathrm{D}$ model of the surface of the RP primary circuit. The algorithm is flexible and easily adaptable to any pressurized water reactor plant.

\section{Leading edge arrival time determination}

One of the key factors in localizing the source of the acoustic anomaly is determining the time of arrival (TOA) of the impact wave for each sensor that recorded the effect. There are several approaches to the TOA determination. One method is to approximate the root-meansquare (RMS) value of the signal by means of a piecewise smooth function:

$$
F(t)= \begin{cases}F_{n}(t)=a, & t<t_{T O A}, \\ F_{S}(t)=k t+b, & t_{T O A}<t<t_{\text {max }},\end{cases}
$$

where $F_{n}(t)$ is the constant value approximating the background noise; $F_{S}(t)$ is the linear function approximating the leading edge in the interval from the TOA $\left(t_{T O A}\right)$ to the RMS maximum time $\left(t_{\max }\right)$, with $F_{n}\left(t_{T O A}\right)=$ $F_{S}\left(t_{T O A}\right)=F\left(t_{T O A}\right)$.

Another widespread approach is based on statistical sequential analysis methods that are used to detect the acoustic effect. These include the Wald sequential probability ratio test (WSPRT) and the CUSUM test (Page 1961). In (Liu Mingzhou et al. 2017), a method is proposed for determining the impact signal TOA based on the Hilbert-Huang transform (HHT) and the Akaike information criterion (AIC).

To determine the TOA, the present work involves the method for detecting changes in the parameters of a random process. The original signal is pre-filtered (Maksimov et al. 2018). A non-stationary random process $y=\left\{y_{t 1}, \ldots, y_{t N}\right\}$ is considered. It is assumed that the signal $y$ is piecewise stationary. At some point in time $t_{T O A}$ the signal dispersion changes dramatically. This point in time is called a 'change point' Assessing the $t_{T O A}$ change point 
is the task of finding the minimum of the objective function (Truong et al. 2018, Lavielle 2005). Since it is necessary to split the signal into only two parts, the objective function can be defined as

$$
F\left(t_{T O A}\right)=c\left(y_{t_{1} \ldots t_{T O A}}\right)+c\left(y_{t_{T O A} \ldots t_{N}}\right),
$$

where $c\left(y_{t i \ldots t i j}\right)$ is the function that measures the uniformity (stability of parameters) of the signal part $y_{i \ldots .}$.

The function $c\left(y_{t i \ldots t j}\right)$ for the signal dispersion change problem can be defined as

$$
c \sigma^{2}\left(y_{i \ldots j}\right)=\left|y_{i \ldots . j}\right| \times \log \sigma_{i \ldots j}^{2},
$$

where $\left\lfloor y_{i \ldots j} \mid\right.$ is the length of the signal part; $\sigma^{2}{ }_{i \ldots j}$ is the signal part dispersion. The values of the objective function are expected to be low for uniform parts of the signals and large with significant dispersion changes. Therefore, the TOA of the impact wave is defined as follows:

$$
t_{T O A}=\arg \min F\left(t_{T O A}\right) .
$$

This method is illustrated in Fig. 1. If there is an acoustic effect (Fig. 1a), the objective function has a single pronounced minimum, which corresponds to the VP. In the case of a signal exclusively of background noise (Fig. 1b), the values of the objective function are stationary and a global minimum is not observed.

\section{Background and assumptions of the localization method}

When loose parts collide with the surface of the RP equipment, an acoustic impact wave is generated. It is assumed that it spreads in the RP equipment material every which way at an equal velocity, which does not depend on the location of the source and direction. As a result, the wave travel time between two points on the surface is uniquely determined by the length of the shortest path along the surface between these points. Therefore, the time between the impact moment and its detection by the sensor depends on the distance and wave velocity.

To localize the anomaly in a linear section, it is enough to have signals from two sensors that recorded the burst. For a plane or surface, which can be represented as a plane, the TDOA pairs of sensors determine the geometric location of the points of the source possible position. To accurately determine the impact site, a signal
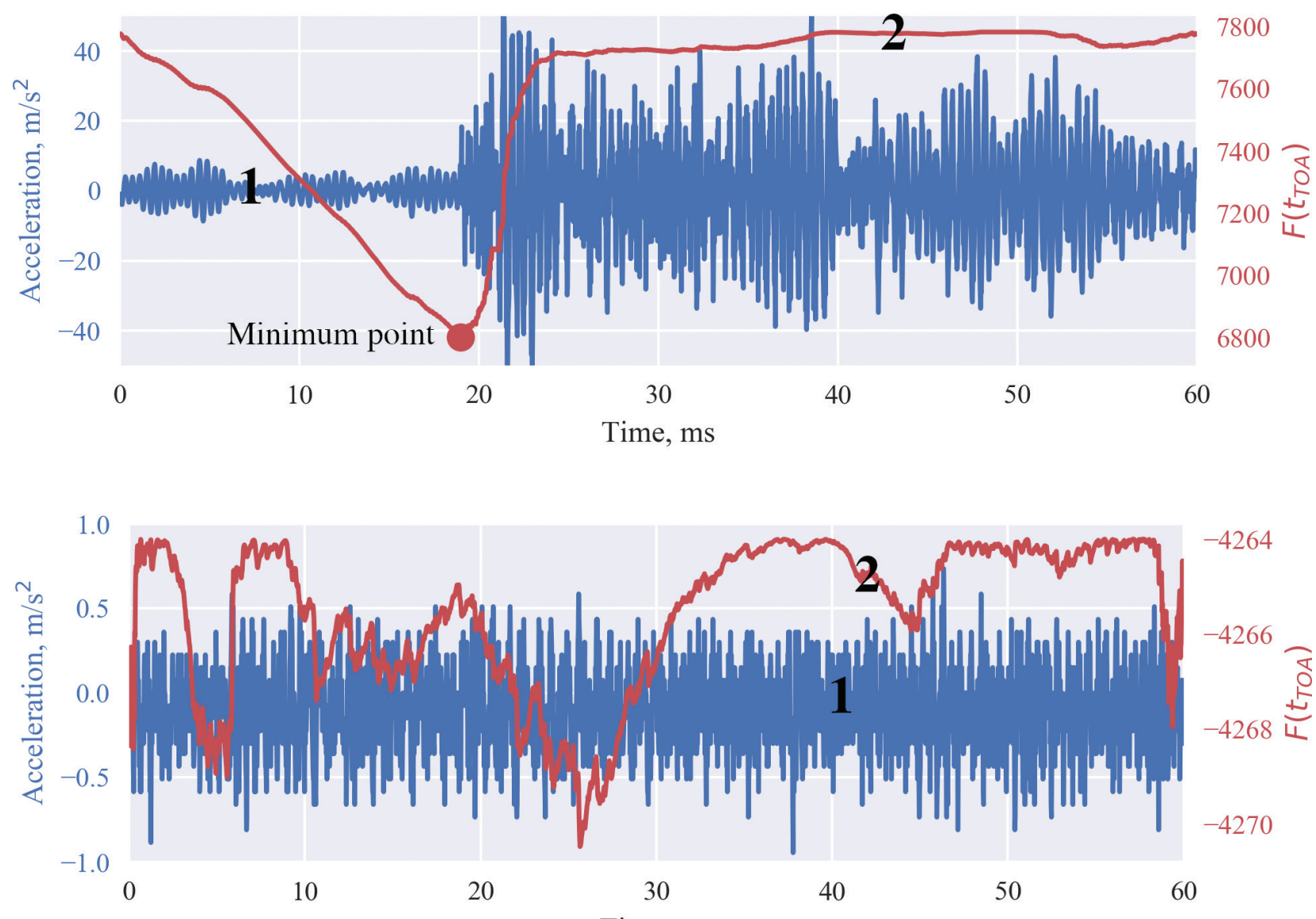

Time, ms

Figure 1. Estimation of the time of arrival of the acoustic signal: a) with an acoustic effect; b) without an acoustic effect (1- signal, 2 - objective function). 
of the third sensor is required. Then the location of the impact wave source is defined as the center of the circle passing through the coordinate of the sensor installation, corresponding to the sensor that was the first to record the impact wave, and tangent to the other two, with the radius $\Delta t_{1 i} \times V$, where $\Delta t_{1 i}$ is the TDOA of the acoustic wave between the sensor that was the first to record the impact and $i$-th sensor with respect to the time of arrival; $V$ is the velocity of sound in the medium (Fig. 2).

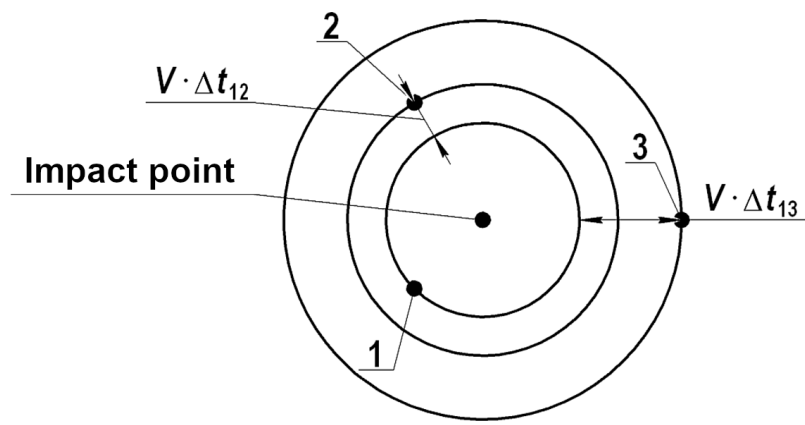

Figure 2. Graphic representation of the TDOA-based acoustic anomaly localization method: 1,2,3 are the sensor sensors numbered according to the time of arrival.

The average wave velocity, considered in the target frequency range, is of the order of $2500 \mathrm{~m} / \mathrm{s}$ (Szappanos et al. 1999). This velocity is used to calculate the coordinate of the impact site when the acoustic effect is recorded by no more than three sensors. When the effect is recorded by four or more sensors, the wave velocity is calculated along with the coordinate of the impact site.

\section{Data preparation}

To obtain the coordinate of the acoustic anomaly source localization in real time, it is necessary to prepare data for the calculation. The main data is taken from the RP and LPMS design documentation:

- a geometric model of the RP surface; and

- positions of the sensors on the RP equipment.

The geometric model is the shape of the boundaries of the pipelines and the main equipment of the $\mathrm{MCC}$, i.e., the surface along which an acoustic wave travels. The positions of the sensors are the fixed sites in which the sensors are installed. They are described by coordinates on the geometric model.

The data preparation stage includes as follows:

1. Selection of control points on the geometric surface (Fig. 3). These points characterize potential impact sites. To facilitate further calculations, they are selected as vertices of a uniformly triangulated surface (grid). The higher the resolution of the grid (the number of vertices), the smaller the error in determining the impact site. On the other hand, with a fairly small partition, the resources expended in memory and processor time during the algorithm operation increase. As a result, we obtain the control points $C_{i}, i=1, \ldots$, $M$, where $M$ is the number of control points.

2. Binding of sensor positions to the nearest control points. The coordinates of the sensors on the RP

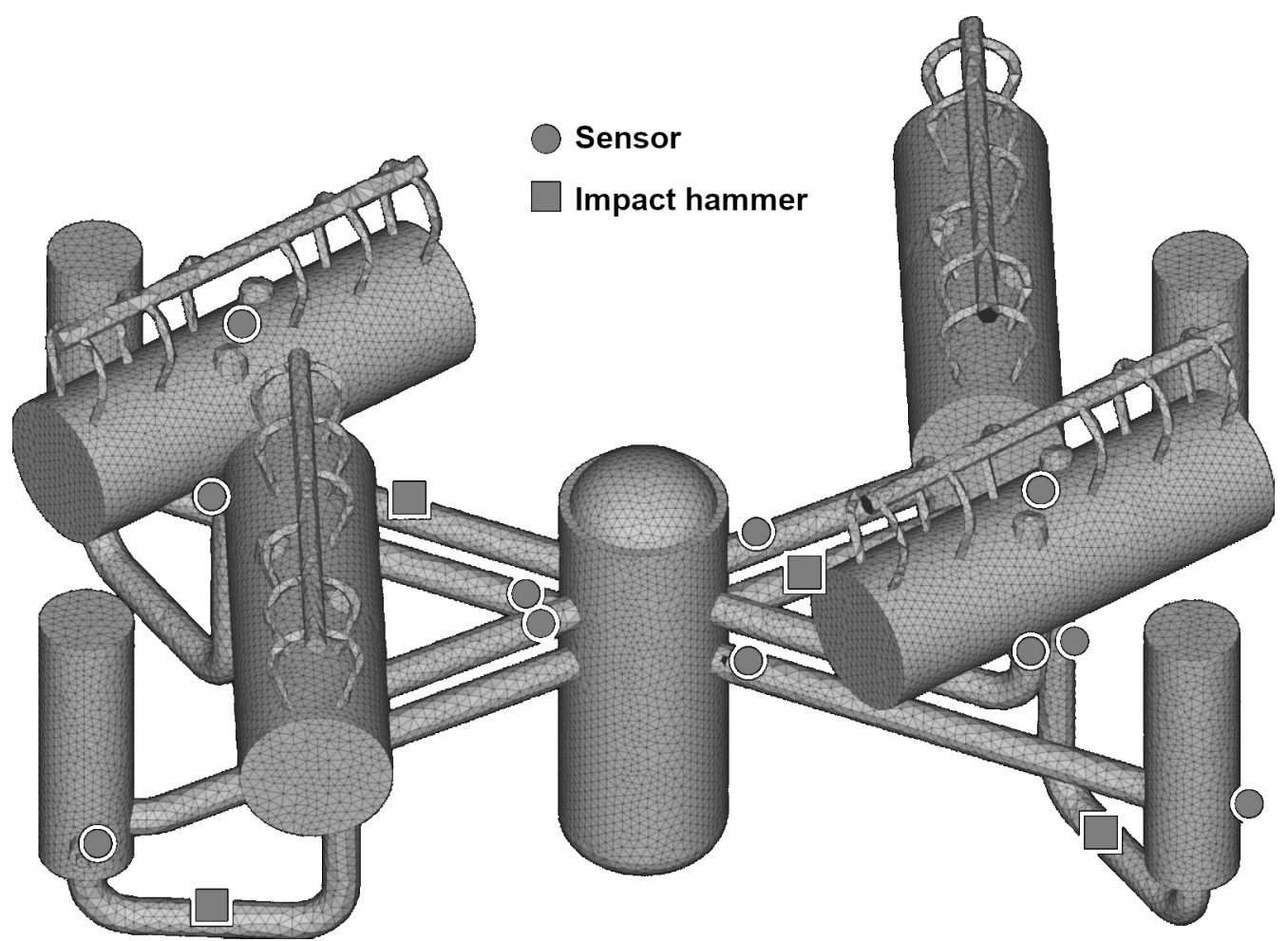

Figure 3. Geometric model of the RP surface and the location of the sensors and hammers. 
equipment are compared with the control points. Triangulation of the surface should be carried out in such a way that the control points of the grid correspond to the coordinates of the sensors. As a result, we obtain the positions of the sensors $S_{i}, i=1, \ldots, N$, where $N$ is the number of the sensors.

3. Calculation of the shortest distances between the control points and sensors using Dijkstra's algorithm (Dijkstra 1959) to find the minimum path in a weighted graph. The control points are considered nodes of the graph, and the lengths of the edges of the grid are weights of the graph. Dijkstra's algorithm is easy to use, but the result is sensitive to the quality of the surface partition. To improve the results, it is necessary to produce a uniform triangulation of the surface, where the grid edges have approximately the same length.

As a result, we obtain the matrix $\mathbf{D}$ dimensioned $[M \times$ $N]$ with elements

$$
D_{i j}=\operatorname{dist}\left(C_{i}, S_{j}\right)
$$

where $C_{i}$ is the $i$-th control point; $S_{j}$ is the $j$-th sensor; dis$\mathrm{t}\left(C_{i}, S_{j}\right)$ is the the function of calculating the shortest distance over the geometric model surface.

\section{Localization algorithm}

The localization algorithm is performed every time an event associated with an unknown acoustic anomaly and (potentially) the presence of loose parts in the primary circuit is recorded. The input to the algorithm includes:

- $\quad$ the vector of the TDOAs of the acoustic wave $\Delta t$ to each sensor $S_{i}$

$$
\Delta t_{i}=T O A_{i}-\min (T O A),
$$

where $T O A_{i}$ is the time of arrival of the acoustic wave;

- the distance matrix D calculated by the formula (5);

- the initial value of the acoustic wave velocity in the $\mathrm{RP}$ equipment material, which is assumed to be equal to $2500 \mathrm{~m} / \mathrm{s}$.

The values of the TDOA vector represent a vector, the size of which corresponds to the number of sensors $N$. In cases when the time of arrival was not determined on the sensor, the TDOA value remains undetermined. The numbers of the sensors, in which the TDOA value was determined, form the set $K$.

The algorithm for calculating the coordinate of the acoustic anomaly occurrence includes as follows:

1. For each control point $C_{i}, i=1, \ldots, M$ the vector $r_{i}$ is calculated:

$$
r_{i j}=D_{i j}-\Delta t_{j} \times V,
$$

where $V$ is the initial approximation of the acoustic wave velocity; $j \in K$ are the sensor numbers where the TDOA is determined.

2. For each vector $r_{i}, i=1, \ldots, M$ the functional value is calculated with the exception of empty values:

$$
\sigma_{i}=\frac{1}{|K|} \sum_{j \in K}\left(r_{i j}-r_{i}^{m}\right)^{2} .
$$

3. The control point $C_{i}$ is found at which the value of $\sigma_{i}$ is minimal:

$$
i=\arg \min (\sigma) .
$$

4. For the obtained control point $C_{i}$, the acoustic wave velocity $V_{j}$ to the sensor $S_{j}$ is re-calculated:

$$
V_{j}=\left(D_{i j}-D_{i j 0}\right) / \Delta t_{j},
$$

where $D_{i j 0}$ is the distance from $C_{i}$ to the sensor $S_{j 0}$ that was the first to record the impact with respect to the time of arrival; $j \in K$ are the sensor numbers where the TDOA is determined.

5. For the calculated velocities $V_{j}$, the average wave velocity is calculated

$$
V^{m}=\frac{1}{|K|} \sum_{j \in K} V_{j}
$$

6. The calculated average velocity $V^{m}$ is compared with the initialized velocity $V$. If the velocity values coinci$\mathrm{de}$, then the algorithm is completed. If the values differ, the velocity $V$ is taken equal to $V^{m}$, and the algorithm is repeated until the velocities $V$ and $V^{m}$ coincide.

\section{Results}

As a result of the algorithm implementation, the coordinate of the acoustic anomaly source $C$ and the average acoustic wave velocity $V^{m}$ are determined. To estimate the accuracy of determining the source localization, impacts of impact hammers (IH) located on the RP pipelines were considered (the arrangement of sensors and IHs is shown in Fig. 3). Sensors are located on each RCPS, hot and cold SG headers, SG vessel, as well as reactor inlet and outlet nozzles. The IH produced an energy impact of $\sim 1.8 \mathrm{~J}$.

The geometric RP model in the form of a grid contained 30004 vertices (control points). The average distance between two control points was $30 \mathrm{~cm}$.

In total, 143 IM impacts were made on different loops. The impacts were made under different operating modes 
Table 1. Experimental results.

\begin{tabular}{lccccc}
\hline Loop No. & Location of IHs & Number of impacts & $\begin{array}{c}\text { Average localization error, } \\
\mathbf{m m}\end{array}$ & $\begin{array}{c}\text { Average acoustic wave } \\
\text { velocity, } \mathbf{m} / \mathbf{s}\end{array}$ & $\begin{array}{c}\text { Distance to the nearest sensor, } \\
\text { mm }\end{array}$ \\
\hline 1 & & 29 & 624 & 2241 & 8803 \\
2 & U-shaped bend & 38 & 708 & 2351 & 5998 \\
3 & Pipeline cold patch & 38 & 473 & 2494 & 2161 \\
4 & Pipeline hot patch & 38 & 768 & 3769 & 8654 \\
\hline
\end{tabular}

of the power unit, and, accordingly, background acoustic noise was different, which affected the accuracy of determining the time of arrival. The experimental results are given in Table 1.

Provided that the average cell size was $300 \mathrm{~mm}$, the average localization error was $600 \mathrm{~mm}$. As the distance from the impact site to the nearest sensor becomes longer, the error increases. The main error is associated with determining the time differences of arrival of the impact wave. The grid edge size introduces a constant error proportional to its length. Nevertheless, the deviation values are completely acceptable for practical use, and the developed algorithm can be used to estimate the acoustic anomaly source localization.

\section{References}

- Choi Y-C, Park J-H, Choi K-S (2011) An Impact Source Localization Technique for a Nuclear Power Plant by Using Sensors of Different Types. ISA Transactions 50 (1): 111-118. https://doi.org/10.1016/j. isatra.2010.08.004

- Dijkstra EW (1959) A Note on Two Problems in Connexion with Graphs. Numerische Mathematik 1(1): 269-271. https://doi. org/10.1007/BF01386390

- Figedy S, Oksa G (2005) Modern Methods of Signal Processing in the Loose Part Monitoring System. Progress in Nuclear Energy 46(3-4): 253-267. https://doi.org/10.1016/j.pnucene.2005.03.008

- IEC 60988 Nuclear power plants (2009) Instrumentation important to safety. Acoustic monitoring systems for detection of loose parts: Characteristics, design criteria and operational procedures. International Electrotechnical Commission, 75 pp.

- ISO 13379-1-2015 (2015) Condition monitoring and diagnostics of machines - Data interpretation and diagnostics techniques Part 1: General guidelines. Moscow. Standartinform Publ., 33 pp. [in Russian]

- Ki HI, Seong-In M, Soon-Woo H (2017) ANN Based Localization of Metal Ball Impacts on Reactor Pressure Boundary Structure. Transactions of the Korean Nuclear Society. Autumn Meeting Gyeongju, Korea, October 26-27, 3 pp. https://www.kns.org/files/pre_paper/38/17A-554***.pdf [accessed 19.02.2019]

- Lavielle M (2005) Using Penalized Contrasts for the ChangePoint Problem. Signal Processing 85(8): 1501-1510. https://doi. org/10.1016/j.sigpro.2005.01.012

- Liska J, Kunkel S (2017) Localization of Loose Part Impacts on the General 3D-surface of the Nuclear Power Plant Coolant Circuit

\section{Conclusions}

The authors propose an algorithm for localizing the acoustic anomaly source on the surface of the RP equipment along the triangulated surface of a 3D model of the coolant circulation circuit.

The algorithm consists of two parts. Data preparation is performed once for each power unit. Then, when an event is recorded in real time, the second part is executed, where the impact site and the average acoustic wave velocity are determined.

The analysis of the experimental data showed that the average error in the impact source localization is $\sim 600 \mathrm{~mm}$, which makes possible the practical application of the developed method.

Components. Progress in Nuclear Energy 99: 140-146. https://doi. org/10.1016/j.pnucene.2017.05.004

- Mingzhou L, Jiangxin Y, Yanpeng C, Weinan F, Yanlong C (2017) A New Method for Arrival Time Determination of Impact Signal Based on HHT and AIC. Mechanical Systems and Signal Processing 86: 177-187. https://doi.org/10.1016/j.ymssp.2016.10.003

- Maksimov IV, Pavelko VI, Perevezentsev VV, Trykov EL (2018) Valid Signal Isolation Method for Loose Parts Monitoring System in the Main Circulation Circuit of VVER Reactor. Vestnik MGTU im. N.E. Baumana. Ser.: Priborostroenie [Bulletin of Bauman MSTU. Ser.: Instrument Engineering] 118: 4-15. [in Russian] https://doi. org/10.18698/0236-3933-2018-1-4-15

- Olma B (1985) Source Location and Mass Estimation in Loose Parts Monitoring of LWR. Progress in Nuclear Energy 15: 583-594. https://doi.org/10.1016/0149-1970(85)90086-1

- Operation and Maintenance of Nuclear Power Plants (2015) Part 12. Loose Part Monitoring. American Society of Mechanical Engineers (ASME), 523 pp.

- Page ES (1961) Cumulative Sum Charts. Technometrics 3(1): 1-9. https://doi.org/10.1080/00401706.1961.10489922

- Park J-H, Kim Y-H (2006) Impact Source Localization on an Elastic Plate in a Noisy Environment. Measurement Science and Technology 17(10): 2757-2766. https://doi.org/10.1088/0957-0233/17/10/030

- Regulatory Guide 1.133 (1981) Loose Part Detection Program for the Primary System of Light-Water Cooled Reactors: tech. rep. U.S. Nuclear Regulatory Commission, 6 pp.

- Soo KJ, Koo HI, Tak KJ, Joon L (2003) Development of Automatic Algorithm for Localizing Loose Parts with a Steam Genera- 
tor. Nuclear Engineering and Design 219(3): 269-276. https://doi. org/10.1016/S0029-5493(02)00281-9

- Szappanos G, Kiss JJ, Por G, Kiss JM (1999) Analysis of Measurements made by HELPS Loose Part Detection System During Installation and operation Periods. Progress in Nuclear Energy 34(3): 185-193. https://doi.org/10.1016/S0149-1970(98)00004-3

- Truong C, Oudre L, Vayatis NC (2018) A Review of Change Point Detection Methods. https://arxiv.org/abs/1801.00718 [accessed Feb 19, 2019]
- Yong PG, Woo CS, Kwon LC, Choon KK (2006) An Estimation Method for Impact Location of Loose Parts. Progress in Nuclear Energy 48(4): 360-370. https://doi.org/10.1016/j.pnucene.2005.09.012

- Zheng H, Cao Y, Yang J (2008) A Method for Estimating Impact Location of Loose Part Using HHT. Proc. of the Fourth International Symposium on Precision Mechanical Measurements. (Fei Y, Fan K-C, Lu R, Eds.) SPIE, 6 pp. https://doi.org/10.1117/12.819732 